\title{
Research on Influencing Factors of Green Innovation of New Energy Vehicles Based on Bayesian Networks
}

\author{
JiaHui $\mathrm{Li}^{1}$, Qin Liu²*, YingXi Ge ${ }^{1}$ \\ ${ }^{1}$ School of Management, Wuhan University of Technology, Wuhan, 430070, China \\ ${ }^{2}$ School of Enterpreneurship, Wuhan University of Technology, Wuhan, 430070, China
}

\begin{abstract}
This article analyzes the four quasi-measurement levels including industrial development, and collects various data such as government policies, fiscal data, and company patents. This paper analyzes the implementation process of policies, explores the research factors of enterprise innovation ability, and obtains 11 indicators. Based on the Bayesian network constructed with 11 indicators, we use linear regression methods to explore the influencing factors of the green innovation capability of new energy automobile companies, and quantify the degree of influence of each factor. Based on the quantitative results, this paper provides suggestions for government industrial strategic planning, taxation policies, government procurement, etc., and provides a reference for the development of new energy automobile enterprises' industry and technology.
\end{abstract}

\section{Introduction}

Nowadays, facing the severe situation of tightening resource constraints, serious environmental pollution, and degradation of the ecosystem, people have gradually begun to establish the concept of ecological civilization that respects nature, conforms to nature, and protects nature, and follows the path of sustainable development. Under the pressure of energy and environmental protection, new energy vehicles will undoubtedly become the development direction of future automobiles. If new energy vehicles develop rapidly, based on the 140 million Chinese car ownership in 2020, 32.29 million tons of oil can be saved, 31.1 million tons of oil can be replaced, and a total of 63.39 million tons of oil can be saved and replaced, which is equivalent to reducing the demand for automobile oil by $22.7 \%$. Before 2020, saving and replacing petroleum will be realized by the development of advanced diesel vehicles and hybrid vehicles. By 2030, the development of new energy vehicles will save 73.06 million tons of oil, replace 91 million tons of oil, and save and replace a total of 164.06 million tons of oil, which is equivalent to reducing automobile oil demand by $41 \%$. By then, biofuels and fuel cells will play an important role in the substitution of automobile petroleum. Since 2008, the Chinese government has launched the "Ten Cities and Thousand Vehicles" energy-saving and new energy vehicle demonstration project, and now requires the indepth implementation of the national strategy for the development of new energy vehicles, promotes the highquality and sustainable development of China's new energy vehicle industry, and accelerates the establishment of a powerful automobile nation. The automotive market has grown rapidly from immature to today. The government plays a pivotal role in this process. This paper is based on refining the indicators of the green innovation influencing factors of new energy vehicles based on the formulation, implementation and effects of public policies. By constructing a Bayesian network model, this paper explores the impact of various factors on the green innovation of new energy vehicle companies. Through data collection, model training, empirical testing, and predictive analysis, we can quantify the influencing factors of green innovation of new energy vehicles, analyze the reasons behind them, and put forward conclusions and suggestions based on the current technological development and policy background.

\section{Literature review}

Since the issue of energy scarcity and new energy vehicle technology has attracted people's attention, many people have studied the technology of new energy vehicle technology. Xu Le et al. used fixed-effects model, double difference method and other methods to test the new energy technology innovation effect of key industrial policies and their mechanism from the demand side, and concluded that different levels of policies have different effects on different regions. [1] Zang Chongchong analyzed the relationship between government subsidies, corporate R\&D investment, and green technology innovation performance, and analyzed the relationship between government and green innovation from the perspective of direct subsidies and tax incentives. [2] Peng Siyu collects and sorts out the new energy vehicle policy data issued at the national level, and uses content analysis to analyze the policy text. From the three stages of

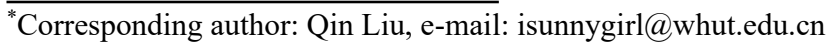


initiation, promotion, and development, it is divided into three categories: supply type, demand type and environmental type. A comparative analysis of the automobile industry policies of the two countries. [3] From the perspective of industry pollution heterogeneity, Zhu Dongbo theoretically analyzes the mechanism of environmental regulation and technological innovation on the green transformation of industrial structure, constructs a dynamic panel model, uses provincial panel data and systematic GMM methods, etc., to strengthen regional environmental regulations Policy recommendations are made in terms of policy regulation and speeding up the process of technological green innovation. [4] Dong Zhiqing and others selected panel data from 154 prefecture-level cities in China, used principal component analysis to evaluate the economic freedom of each city, and explored the relationship between economic freedom and green technological innovation and its causes. Corresponding policy recommendations were put forward from the urban green technological innovation and its constraints. [5] Based on the input-output data of industrial enterprises, Tian Hongbin and others used the GML index to measure the efficiency of green innovation and its decomposition, and analyzed the impact of FDI inflows on the efficiency of green innovation in the three cases of command control, investment and cost environmental regulations. influences. It is concluded that the combination of appropriate environmental regulations and scientific FDI introduction policies can effectively improve the efficiency of green innovation. [6] Zhang Shuai took the supply chain collaboration of corporate green innovation as the research object, and discussed the supply chain collaboration mechanism of corporate green

Table 1. Green Innovation Evaluation Syste innovation. The coordination mechanism is designed by game theory method. [7]

In the past research, few people used Bayesian theory and other statistical methods to conduct research, and they did not explore some of the factors. Based on Bayesian network theory, this paper can better analyze the relationship between factors, and use statistical methods to quantify the relationship between low analysis policies and other factors and green innovation of new energy vehicles.

\section{Data and Methodology}

\subsection{Index system construction}

The rapid development of new energy vehicles has largely benefited from government policy encouragement and promotion. In addition, the overall industry standards of the relevant technical level also determine the bottleneck of the development of this new energy vehicle technology. Whether a technology can be recognized by consumers is also related to the publicity of the technology by social media organizations. Based on the actual development of new energy vehicle technology, the number of patents of new energy vehicle companies is used as a standard to measure the innovation ability of the company, and four aspects are selected as the criterion layer: industrial development, technology research and development, application promotion, and environmental protection. Based on the four quasi-measurement layers, a total of 11 indicators are selected as the indicator layers. The specific evaluation system is shown in Table 1.

\begin{tabular}{|c|c|c|c|}
\hline Target layer & Criterion layer & Index layer & Data Sources \\
\hline \multirow{11}{*}{ Enterprise Innovation } & \multirow{4}{*}{ Industrial Development } & Financial Subsidy & Government agency \\
\hline & & Tax Incentives & Government agency \\
\hline & & Strategic Planning & Government agency \\
\hline & & Intellectual Property & Government agency \\
\hline & \multirow[t]{2}{*}{ Technology R \& D } & Battery Technology & $\begin{array}{l}\text { Related companies and } \\
\text { manufacturers }\end{array}$ \\
\hline & & Charging Technology & $\begin{array}{l}\text { Related companies and } \\
\text { manufacturers }\end{array}$ \\
\hline & \multirow{4}{*}{ Application promotion } & $\begin{array}{l}\text { Government } \\
\text { Procurement }\end{array}$ & Government agency \\
\hline & & Propaganda Power & Social media agency \\
\hline & & Infrastructure & Government agency \\
\hline & & Access Rules & Government agency \\
\hline & Environmental protection & $\begin{array}{c}\text { Environmental } \\
\text { Regulation }\end{array}$ & Government agency \\
\hline
\end{tabular}

\subsection{Data processing and methods}

The theoretical basis of Bayesian networks is derived from Bayes' theorem.

$$
P\left(H_{i} \mid E\right)=\frac{P\left(E \mid H_{i}\right) P\left(H_{i}\right)}{\sum_{k=1}^{n} P\left(E \mid H_{k}\right) P\left(H_{k}\right)}
$$

Since the static Bayesian theory cannot dig out the direct correlation between factors, a Bayesian network is constructed and the direct relationship of various indicators is connected, making the model more practical. In this paper, when the structure of the Bayesian network is known but the parameters are unknown, we must use the observed data to estimate the online parameters, so we need to use the maximum likelihood estimation method to supplement the network data.

Given a probability distribution D, assuming its probability density function or probability aggregation 
function is $f_{D}$, and a distribution parameter $\theta$, we can extract a sample $X_{1}, X_{2}, \ldots, X_{n}$ with $n$ values from this distribution, and pass Using $f_{D}$, we can calculate its probability. However, we may not know the value of $\theta$, so we can extract a sample $\mathrm{X}_{1}, \mathrm{X}_{2}, \ldots, \mathrm{X}_{\mathrm{n}}$ with $\mathrm{n}$ values from this distribution, and then use these sampled data to estimate $\theta$.

This article will take the number of patents as a measure of the innovation capability of a company. By analyzing the relationship between various indicators, the Bayesian network structure as shown in Figure 1 is constructed. After constructing the model, collect various indicator data of mainstream new energy automobile companies from the data source on a monthly basis since 2014. After data processing such as data cleaning and data filling, it is standardized, and then the value of each indicator is graded, divided into high $(\mathrm{H})$ and low $(\mathrm{L})$ levels, and substituted into the Bayesian network for maximum likelihood estimation Fitting training, get the probability of each node, and then provide the basis for the next step of predictive analysis.

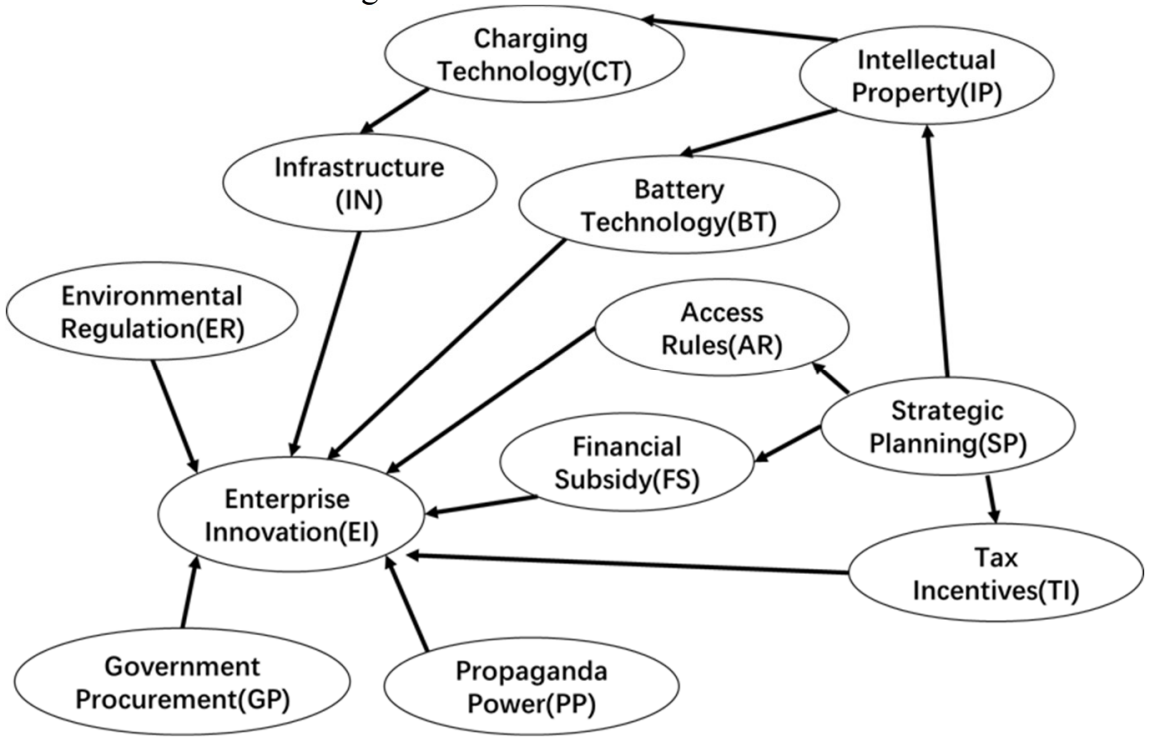

Figure 1. Bayesian network structure of green innovation for new energy vehicles

\section{Empirical Analysis}

After collecting and cleaning the patent data of each category of the top five new energy vehicle companies. After bringing these data into the Bayesian network, the probability assignment of each node of the Bayesian network is completed. This article considers the different permutations and combinations of various factors, and obtains the innovation incentive effect through traversal. The ranking of the probability values of the innovation nodes is shown in the table 2.

Table 2. Sorting of probability values of EI nodes

\begin{tabular}{cccccccccccc}
\hline FS & TI & SP & IP & BT & CT & GP & PP & IN & AR & ER & P(EI=H) \\
\hline L & L & H & H & L & H & H & L & L & H & L & 0.647059 \\
L & L & H & H & L & L & H & L & L & H & L & 0.647059 \\
L & L & L & H & L & H & H & L & L & H & L & 0.647059 \\
L & L & H & L & L & H & H & L & L & H & L & 0.647059 \\
L & H & L & H & H & L & H & H & H & L & H & 0.622642 \\
L & H & L & H & H & H & H & H & H & L & H & 0.622642 \\
L & H & H & H & H & L & H & H & H & L & H & 0.622642 \\
H & L & H & H & L & H & L & L & L & H & L & 0.583333 \\
H & L & L & H & L & H & L & L & L & H & L & 0.583333 \\
H & L & L & H & L & L & L & L & L & H & L & 0.583333 \\
\hline
\end{tabular}

Strategic Planning and Government Procurement has a great influence on the probability of EI nodes. In order to further study the relationship between them, this paper uses the method of linear regression to obtain the coefficients of each factor in the linear model, and use the coefficients to determine the impact on the EI node, as shown in Table 3. 
Table 3. The coefficients of each factor in the model

\begin{tabular}{cccccc}
\hline & $\begin{array}{c}\text { Unstandardized } \\
\text { Coefficients } \\
\text { B }\end{array}$ & Std. Error & $\begin{array}{c}\text { Standardized } \\
\text { Coefficients } \\
\text { Beta }\end{array}$ & $\mathrm{t}$ & Sig. \\
\hline (Constant) & 0.568 & 0.016 & & 34.514 & 0.000 \\
FS & -0.029 & 0.008 & -0.255 & -3.725 & 0.000 \\
TI & 0.124 & 0.016 & 0.980 & 7.870 & 0.000 \\
SP & -0.009 & 0.008 & -0.075 & -1.095 & 0.279 \\
IP & -0.009 & 0.008 & -0.075 & -1.095 & 0.279 \\
BT & -0.030 & 0.011 & -0.276 & -2.811 & 0.007 \\
CT & -0.008 & 0.007 & -0.075 & -1.184 & 0.242 \\
GP & 0.030 & 0.011 & 0.242 & 2.813 & 0.007 \\
PP & -0.022 & 0.012 & -0.200 & -1.846 & 0.070 \\
IN & -0.018 & 0.010 & -0.163 & -1.798 & 0.078 \\
AR & 0.039 & 0.011 & 0.363 & 3.446 & 0.001 \\
ER & -0.039 & 0.011 & -0.363 & -3.594 & 0.001 \\
\hline
\end{tabular}

The largest coefficient is TI. It can be seen that Tax Incentives has a greater impact on the green innovation of new energy vehicle companies. In addition, Government Procurement and Access Rules also have a certain impact on corporate innovation.

\section{Conclusion}

Based on the results of the previous analysis, combined with the current development of the new energy automobile industry, we draw conclusions and recommendations as follows:

(1) The government's strategic planning for the new energy vehicle industry plays a decisive role in the development of new energy vehicle technology to a certain extent. Therefore, the government should accurately position the industrial plan and formulate a reasonable industrial plan based on the actual situation.

(2) Government procurement can stimulate new energy vehicle companies to actively bid, and the development of procurement specifications can stimulate the technological development of new energy vehicle companies. Under the current situation that electric public transportation has become widespread, the public transportation should be updated in time to promote new energy and the development of automotive technology.

(3) Compared with direct subsidies, tax incentives have a greater positive effect on the technological innovation of new energy companies. The government should further formulate detailed and reasonable preferential policies in accordance with the actual development of the industry in terms of tax policies.

(4) By improving the detail of the access rules, the company's green innovation capabilities can be further strengthened.

\section{Acknowledgment}

This work is supported by the National Social Science Foundation of China (No. 19BSH105), the Fundamental
Research Funds for the Central Universities (WUT: 2020VI028), and National innovation and entrepreneurship training program for college students S202010497038 and 202010497018

\section{References:}

1. Xu Le, Zhao Lingdi. New energy technology innovation effect of key industrial policy [J]. Resources Science,2019,41(01):113-131.

2. Zang Chongchong. Research on the Correlation between Government Subsidy and Enterprise Green Technology Innovation [D]. North China University of Technology,2018.

3. Siyu Peng. New Energy Vehicle Development Policy and Green Innovation of Automobile Enterprises [D]. Huazhong University of Science \& Technology,2018.

4. Zhu Dongbo. Environmental Regulation, Technology Innovation and Industrial Structure Green Transformation in China $[\mathrm{J}]$. Journal of Industrial Technological Economics,2020,39(10):57-64.

5. Dong Zhiqing, Tan Yusong. An Empirical Study on the Relationship between Economic Freedom andGreen Technology Innovation and Its Causes [J]. Social Science Front,2020(09):99-109.

6. TIAN Hong-bin, HAO Wen-wen. FDI 、 Environmental Regulation and Green Innovation Efficiency [J]. China Soft Science,2020(08):174-183.

7. Shuai Zhang. Research on Supply Chain Synergetic Mechanism of Green Innovation in Enterprises [D]. Wuhan University of Technology,2018. 